\title{
On the Dynamics of Asymmetric Games
}

\author{
ANdrea Gaunfrsdorffr, Iosff Hofraufr, and Kari. Sigmund \\ Institut für Mathematik der Universität Wien, \\ and IIASA, Laxenburg, Austria \\ Received August 16, 1989
}

\begin{abstract}
A game dynamical analysis of a simple asymmetric game (two roles with two alternatives each) shows that an interesting class of "semi-stable" heteroclinic cycles leading to a highly unpredictable behaviour can occur in a robust way. Biological examples related to conflicts over ownership and parental investment are analysed. (C) 1991 Academic Press, Inc.
\end{abstract}

\section{INTRODUCTION}

Even in the artificial world of "fair" parlour games and sporting encounters, asymmetries between the contestant's roles are frequent. Some rules are meant to reduce this asymmetry (toss up a coin to decide who moves first; play a return match) but others emphasise it (a draw favours the incumbent champion; the nation organizing the world soccer cup automatically qualifies). In nature, the role of asymmetries is much more pronounced still, and soon after the introduction of game theory in the study of biological contests, a series of papers underlined the special "logic of asymmetric contests" and drew attention to conflicts with in-built asymmetries like those between owner and intruder, weaker and stronger contestant, male and female, parent and offspring, queen and worker, prey and predator, etc. In Maynard Smith's book on "Evolution and the Theory of Games" (1982), three chapters are devoted to asymmetric games, and a rough census of its list of references seems to show that the majority of conflicts studied by sociobiologists exhibits asymmetries.

In contrast to this, the study of the dynamics of asymmetric games has lagged considerably behind that of the symmetric case. Of the few papers, most have investigated the case of separate populations (Taylor, 1979; Schuster et al., 1981). This is appropriate for coevolutionary games between predator and prey or host and parasite, but hardly so for games between owner and intruder or parent and offspring, where one individual will find itself sometimes in one role and sometimes in the other. It is also plausible that for male-female or worker-queen conflicts, the genetic 
programs for the two roles are linked. In any case, most of the static game theoretical models assume conditional strategies (for example: if male, be a philanderer; if female, be coy). In the present paper we discuss the corresponding dynamical features for the simplest case (two roles with two strategies each).

In Section 2 we describe these dynamics; in Section 3 we apply this to some examples of asymmetric games in biology (battling spiders, bluffing shrimps, and coy birds); and in Section 4 we add a recombination term which reduces the dynamic degeneracy. Of special interest are games with cyclic structure, which exhibit a novel type of "semi-stable" heteroclinic cycle and a "zip"-like bifurcation from stability to instability along a line of equilibria. Proofs are deferred to the Appendix.

\section{THE MODEL}

In the simplest possible case, therc arc two roles I and II with two strategies each: $\mathbf{e}_{1}$ and $\mathbf{e}_{2}$ for $\mathbf{I}$ and $\mathbf{f}_{1}$ and $\mathbf{f}_{2}$ for II. Any individual will find itself with probability $p$ in role I and $1-p$ in role II. (This role can change during its life history, e.g., child and parent or owner and intruder, or it can stick for life, like male and female in most cases.) Following Maynard Smith (1982), we assume that an individual's role is independent of its strategy. Within the game considered, individuals in one role interact only with those in the other role; interactions occur in random pairs.

The payoff for role I (resp. II) is given by a $2 \times 2$ matrix $E=\left(E_{i j}\right)_{i, j=1,2}$ (resp. F): $E_{12}$, for example, is the expected payoff for an $\mathbf{e}_{1}$-strategist meeting an $\mathbf{f}_{2}$-player, etc. Thus we are dealing with bimatrix games.

The population will consist of four "behavioural" types: $\mathbf{G}_{1}=\mathbf{e}_{1} \mathbf{f}_{1}$ (i.e., play $\mathbf{e}_{1}$ if in role I and $\mathbf{f}_{1}$ if in role II), $\mathbf{G}_{2}=\mathbf{e}_{2} \mathbf{f}_{1}, \mathbf{G}_{3}=\mathbf{e}_{1} \mathbf{f}_{2}$, and $\mathbf{G}_{4}=\mathbf{e}_{2} \mathbf{f}_{2}$, with frequencies $x_{1}$ to $x_{4}$, respectively. The state of the population is given by a point in the simplex

$$
S_{4}=\left\{\mathbf{x}=\left(x_{1}, \ldots, x_{4}\right): x_{i} \geqslant 0, x_{1}+\cdots+x_{4}=1\right\} .
$$

Let $\Gamma$ be the sequence of four edges connecting the corners $\mathbf{G}_{1}$ to $\mathbf{G}_{2}$ to $\mathbf{G}_{4}$ to $\mathbf{G}_{3}$ and back to $\mathbf{G}_{1}$ again. Each edge connects two types using the same option in one role and different ones in the other. Generally, one of the two alternatives is the better one, and we orient the edge accordingly. This leads essentially to the following possible orientations of $\Gamma$ (see Fig. 1).

According to a basic result (Selten, 1980), an asymmetric game has no mixed ESS (evolutionarily stable strategy). It is easy to see that type $\mathbf{G}_{j}$ is an ESS if and only if both adjacent edges point towards it. Thus (a) and 


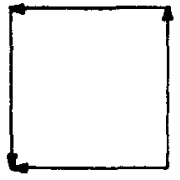

a

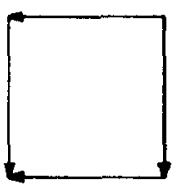

b

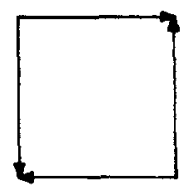

c

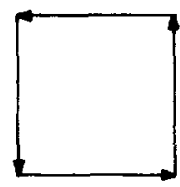

d

FIG. 1. The four possible oricntations of $\Gamma$.

(b) have one ESS and (c) a pair of opposite ESSs while the cyclic structure of (d) allows no ESS at all. We shall see how the dynamics agrees with this static classification.

The payoff-matrix is

$$
M=\left(\begin{array}{llll}
A+a & A+b & B+a & B+b \\
C+a & C+b & D+a & D+b \\
A+c & A+d & B+c & B+d \\
C+c & C+d & D+c & D+d
\end{array}\right),
$$

where

$$
p E=\left(\begin{array}{ll}
A & B \\
C & D
\end{array}\right) \quad \text { and } \quad(1-p) F=\left(\begin{array}{ll}
a & b \\
c & d
\end{array}\right)
$$

For example, a $\mathbf{G}_{2}$-individual is with probability $p$ in role $\mathbf{I}$; its strategy, then, is $\mathrm{e}_{2}$; it interacts only with individuals in role II of which $x_{j}$ are of type $\mathbf{G}_{j}$. Thus its expected payoff is

$$
\left(\text { payoff for } \mathbf{G}_{2} \mid \text { role } \mathbf{I}\right)=x_{1} E_{21}+x_{2} E_{21}+x_{3} E_{22}+x_{4} E_{22} .
$$

With probability $1-p$, the $\mathbf{G}_{2}$-individual is in role II, plays strategy $\mathbf{f}_{1}$, and obtains

$$
\left(\text { payoff for } \mathbf{G}_{2} \mid \text { role II }\right)=x_{1} F_{11}+x_{2} F_{12}+x_{3} F_{11}+x_{4} F_{12} .
$$

The expected payoff for $\mathbf{G}_{2}$ is therefore

$$
p\left(\text { payoff for } \mathbf{G}_{2} \mid \text { role I }\right)+(1-p)\left(\text { payoff for } \mathbf{G}_{2} \mid \text { role II }\right)
$$

which is given by $\sum x_{j} M_{2 j}=(M \mathbf{x})_{2}$.

Thus the game is described now by a single $4 \times 4$ matrix $M$. The payoff is the increment in reproductive value. According to the usual game 
dynamics (Taylor and Jonker, 1978; see also Hofbauer and Sigmund, 1988) we assume that the rate of increase of each type is given by the difference between its payoff and the average payoff in the population. This yields

$$
\dot{x}_{i}=x_{i}\left[(M \mathbf{x})_{i}-\bar{M}\right]
$$

with $\bar{M}=\sum x_{i}(M \mathbf{x})_{i}$. The state space $S_{4}$ and its boundary (consisting of the faces where $x_{i}=0$ ) are invariant. From now on we consider only the restriction of (1) to $S_{4}$. Subtracting $m_{i i}$ from the $i$ th column of $M$ does not affect the dynamics. Hence we may use without restriction of generality the matrix

$$
M=\left(\begin{array}{cccc}
0 & -R & -r & S+s \\
R & 0 & -S-r & s \\
r & -R-s & 0 & S \\
R+r & -s & -S & 0
\end{array}\right)
$$

where $R=C-A, S=B-D, r=c-a$, and $s=b-d$.

One checks immediately that the ratio $x_{1} x_{4} / x_{2} x_{3}$ is an invariant of motion, i.e., remains constant under (1). Each equation $x_{1} x_{4}=K x_{2} x_{3}$ (for $K>0$ ) defines a saddle-shaped surface $W_{K}$ in the interior of the state space $S_{4}$. It is bounded by the four edges belonging to $\Gamma$.

Of special interest is the case $K=1$. In the well-known two-locus, twoalleles equation from populations genetics (see, e.g., Crow and Kimura, 1970 ) the surface $W_{1}$ is called the Wright manifold. In this genetic model $\mathbf{G}_{1}$ to $\mathbf{G}_{4}$ are the gametes, $x_{1}$ to $x_{4}$ are the gamete frequencies, and $x_{1} x_{4}=x_{2} x_{3}$ means that the allele pairs are in linkage equilibrium. In our setup, $x_{1} x_{4}=x_{2} x_{3}$ means that the strategies in role $\mathrm{I}$ and role II are independent. $W_{1}$ divides $S_{4}$ into two halves.

The equilibria of (1) are given by $(M \mathbf{x})_{i}=\bar{M}$ whenever $x_{i}>0$. In int $S_{4}$, this means $(M \mathbf{x})_{1}=\cdots=(M \mathbf{x})_{4}$. Together with $x_{1}+\cdots+x_{4}=1$, this yields a system of four linear equations which is in general of rank 3 . The equations $(M \mathbf{x})_{2}=(M \mathbf{x})_{1}$ and $(M \mathbf{x})_{2}=(M \mathbf{x})_{4}$ imply

$$
x_{1}+x_{2}=\frac{S}{R+S}, \quad x_{1}+x_{3}=\frac{s}{r+s}
$$

if the denominators do not vanish. These two equations determine a line of fixed points, which can be written as

$$
\begin{array}{ll}
x_{i}=m_{i}+\mu & (i=1,4) \\
x_{i}=m_{i}-\mu & (i=2,3)
\end{array}
$$


with

$$
\mathbf{m}=\frac{1}{(R+S)(r+s)}(S s, S r, R s, R r) .
$$

We note that $\mathbf{m}$ satisfies $m_{1} m_{4}=m_{2} m_{3}$ and that the line of fixed points given by (2) intersects int $S_{4}$ if and only $\mathbf{m} \in W_{1}$, i.e., if and only if $R S>0$ and $r s>0$. Thus either all of the invariant surfaces $W_{K}(0<K<\infty)$ contain a fixed point or none do. The $\mu$-values for which (2) then yields a point in int $S_{4}$ are those satisfying

$$
-\min \left(m_{1}, m_{4}\right)<\mu<\min \left(m_{2}, m_{3}\right) .
$$

One can compute the Jacobian and hence the eigenvalues of (1) at the interior equilibria. One eigenvalue is always 0 , of course. We list now a brief classification and deal subsequently with some biologically relevant examples. Essentially, the arrows of the diagrams in Fig. 1 will correspond to orbits of (1) along the edges of $\Gamma$.

(A) No equilibrium in the interior. Then all orbits converge to the boundary, so that the dynamics are reduced to a simple lower dimensional one. Actually, it is easy to check that generically, there exists a single corner of $S_{4}$ which attracts all interior orbits. This corner corresponds to an ESS, and hence we obtain cases (a) or (b) of Fig. 1. Thus the outcome is fixation of a single type. We call this the case of global stability.

(B) A line of equilibria in the interior, and $R r>0$. Each equilibrium is a saddle on the corresponding invariant surface. A surface $S$ containing the equilibria and two corners divides $S_{4}$ into two parts. In each, there is a corner attracting all orbits, while the orbits in $S \cap S_{4}$ converge to interior equilibria. $S$ consists of orbits leading from the repelling corners to the fixed point line (see Fig. 2). This is the case of bistability: up to a set of measure zero, all initial conditions lead to one of two opposite corners. These comers are ESSs. This corresponds to (c) in Fig. 1.

(C) A line of equilibria in the interior, and $R r<0$. This is the cyclic case: $\mathbf{G}_{1}$ beats $\mathbf{G}_{2}$, which beats $\mathbf{G}_{4}$, which beats $\mathbf{G}_{3}$, which in turn beats $\mathbf{G}_{1}$ (or the other way round). The Jacobian at the inner equilibria has a pair of complex eigenvalues, which corresponds to a rotational component on the invariant surface $W_{K}$.

On $W_{1}$, the eigenvalues are purely imaginary. There exists a further invariant of motion, namely

$$
S \log \left(x_{1}+x_{2}\right)+R \log \left(x_{3}+x_{4}\right)-s \log \left(x_{1}+x_{3}\right)-r \log \left(x_{2}+x_{4}\right)
$$

(actually, this is a Hamiltonian on $W_{1}$ ). Thus $W_{1}$ consists of periodic orbits (see Fig. 3), which is also seen from (4) below. For $K>1$ the fixed point 


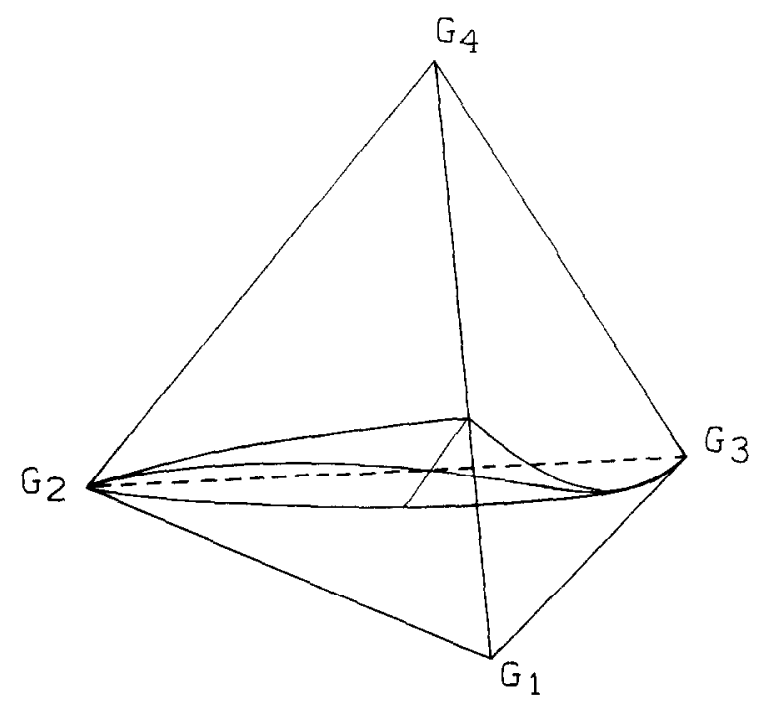

FIG. 2. The simplex is divided into two basins of attraction (with $R=-4, S=-6, r=-2$, $s=-3$ ).

is a spiral sink, and for $K \in(0,1)$ a spiral source (or vice versa), provided $R+S+r+s \neq 0$. Thus if one travels along the line of equilibria, there occurs a degenerate Hopf bifurcation as one crosses $W_{1}$ : stability changes into instability. This is somewhat similar to the zip bifurcation studied by Farkas (1984) in ecological models although there is no parameter here to zip the zipper.

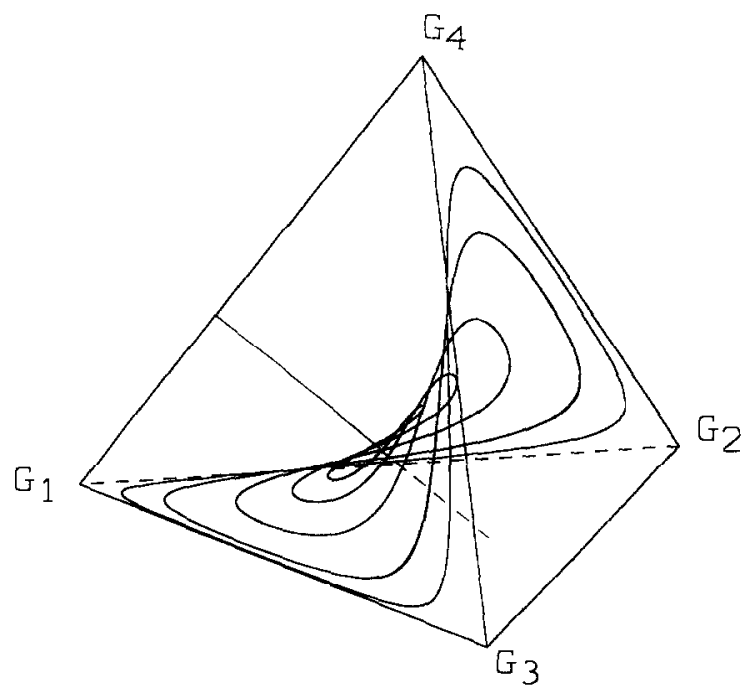

Fig. 3. Orbits on the Wright manifold $W_{1}$ for $R=4, S=6, r=-2, s=-3$. 
We show in the Appendix that off $W_{1}$, there is no periodic orbit. The edges are orbits converging to one corner as $t \rightarrow-\infty$ and to the next one as $t \rightarrow+\infty$. Together, they form a heteroclinic cycle. In one half of int $S_{4}$, all orbits spiral away from the inner equilibria and towards $\Gamma$. In the other half, they spiral away from $\Gamma$ and towards an interior equilibrium.

In the class of all dynamical systems, such a behaviour is structurally unstable to a high degree: (a) the saddle connections-orbits leading from one saddle-type equilibrium to another-correspond to intersections of stable and unstable manifolds which are not transversal, which is a nongeneric situation; (b) the constant of motion foliating the state space into invariant surfaces $W_{K}$ is nongeneric too; (c) the line of equilibria is a degeneracy, since usually equilibria are isolated; (d) the Hopf bifurcation (which leads to no limit cycle) is also degenerate, since at the critical parameter value $K=1$ there occurs a constant of motion. Nevertheless, this heteroclinic cycle which is partly attracting and partly repelling occurs in a perfectly robust way within the dynamics of asymmetric games: a small change in parameters will leave the behaviour unaffected.

The outcome is highly unpredictable. In one-half of the state space, the evolution tends towards an equilibrium with all four types present. However, this equilibrium is only neutrally stable, as it is imbedded within a line of equilibria. Under random fluctuations, the state will drift along this line and eventually enter the other half of the simplex. There, the dynamics will lead towards $\Gamma$. The state will hover close to one corner, then abruptly switch along an edge to the next corner, stay there for a much larger time, switch rapidly (and without exterior cue) to the next one, etc., in a "cycle" with ever increasing period. Since the state is close to $\Gamma$, two or three of the types are present in only a minute amount. Eventually, one of them will be wiped out by a random fluctuation. Then, the cycle is broken and fixation at one of the corners occurs (it is impossible to predict which one). A fortunate sequence of fluctuations (due to mutation or migration for example) can reintroduce some of the missing types, or even all of them; it could even happen that this leads to a state in that half of $S_{4}$ where convergence to an interior fixed point occurs, and the whole evolution is repeated again. But basically, the outcome is fixation of a randomly chosen pure type.

\section{EXAMPLES}

\section{(A) Battling Spiders}

Conflicts between the owner of a territory and an intruder have been analysed by Hammerstein (1981), Maynard Smith (1982), Maynard Smith 
and Parker (1986), and others, and exemplary field studies of territorial fights of funnel web spiders have been carried out by Riechert (1978). Let role I be that of the owner and role II that of the intruder. Both contestants have the option to escalate ( $\mathbf{e}_{1}$ resp. $\mathbf{f}_{1}$ ) or to stick to ritualized fighting. We denote by $-D$ the cost of an injury and by $-T$ that of a drawn-out ritualized conflict. For the owner, the probability of winning is $\frac{1}{2}$ in a ritual fight and $q$ in an escalated battle. By $V_{1}$ and $V_{2}$ we denote the value of the territory for the owner resp. for the intruder (they need not be the same). The payoff matrices are

$$
\begin{aligned}
& E=\left(\begin{array}{cc}
q V_{1}-(1-q) D & V_{1} \\
0 & V_{1} / 2-T
\end{array}\right) \\
& F=\left(\begin{array}{cc}
(1-q) V_{2}-q D & V_{2} \\
0 & V_{2} / 2-T
\end{array}\right) .
\end{aligned}
$$

Then $R=(1-q) D-q V_{1}, \quad r=q D-(1-q) V_{2}, \quad S=V_{1} / 2+T>0, \quad s=$ $V_{2} / 2+T>0$. Interior fixed points exist iff $R>0$ and $r>0$. In this case the fixed points are saddles. We have a bistable case with two ESSs (the bourgeois strategy-owner escalates and intruder flees--and the opposite, paradoxical strategy). As mentioned in Maynard Smith (1982, p. 95), the assumption that roles and strategies are independent is not quite watertight if an animal participates in a series of contests, since the readiness to escalate affects the probability that the animal is an owner in the next contest.

\section{(B) Bluffing Shrimps}

This example has been proposed by Gardner and Morris (1989) to describe the territorial behaviour of a mantis shrimp, which lives and hides in cavities. These crustaceans undergo periodically a stage during which their exoskeleton is renewed. Such newly molten individuals are highly vulnerable to conspecific attacks. Nevertheless, they display sometimes a threatening behaviour towards intruders, leaning out of their cavity and raising their raptorial appendage in a so-called meral spread (a "bluffing" signal similar to the shaking of a fist). If the intruder is in an intermolt stage, it would win an escalated fight, but does not know the actual state of the owner ( $P$ is the probability that it is newly molten).

In the Gardner-Morris example, role $I$ is: owner in a newly molten stage, and role II: intermolt intruder. The owners strategies are $\mathbf{e}_{1}$ (to flee) and $\mathbf{e}_{2}$ (to bluff), while the intruders alternatives are $\mathbf{f}_{1}$ (to attack) or $\mathbf{f}_{2}$ (to withdraw). If $V$ denotes the value of the territory, $-B$ the cost of bluffing (leaning out of the cavity is not without danger) and $-C$ the cost of losing a fight, the payoff matrices are 


$$
\begin{aligned}
& E=\left(\begin{array}{cc}
0 & 0 \\
-B(1-P) & (V-B)(1-P)
\end{array}\right) \\
& F=\left(\begin{array}{cc}
V(1-P)-C P & V(1-P)-C P \\
V(1-P) & 0
\end{array}\right) .
\end{aligned}
$$

This yields $R=-B(1-P)<0, r=C P>0, S=(B-V)(1-P)$ and $s=$ $V(1-P)-C P$, and hence $R+S+r+s=0$ a degenerate case which displays an extra constant of motion (see Appendix). If we assume $B<V$ and $C P<V(1-P)$, so that $S<0$ and $s<0$, there is a line of fixed points in int $S_{4}$. All other orbits in int $S_{4}$ are periodic.

\section{(C) Coy Birds}

A pretty example related to the question of parential investment has been proposed by Dawkins (1976). Some (hypothetical) male birds are faced with the temptation to desert (leaving the female with the task of raising the brood) and to found a new family somewhere else. The counterstrategy of the females is to insist upon a long engagement. It would then be too late in the season, for the male, to start it all over again, and much better to stay and help with the offspring. The two roles are female (I) and male (II); the female can be coy, i.e., insist upon a long engagement before copulation $\left(\mathbf{e}_{1}\right)$, or it can bc fast $\left(\mathbf{e}_{2}\right)$. The male can be a philanderer, i.e., not prepared to put up with a long wait $\left(\mathbf{f}_{1}\right)$, or it can be faithful, i.e., willing to accept a long engagement $\left(\mathbf{f}_{2}\right)$. If $G$ denotes the increase in fitness (for each parent) corresponding to the successful raising of a brood, $-C$ the cost of parental investment (which can be shared, or borne entirely by the female), and $-E$ the cost inflicted by a long engagement on each partner, then the payoffs are given by

$$
\begin{aligned}
& E=\left(\begin{array}{cc}
0 & G-C / 2-E \\
G-C & G-C / 2
\end{array}\right) \\
& F=\left(\begin{array}{cc}
0 & G \\
G-C / 2-E & G-C / 2
\end{array}\right) .
\end{aligned}
$$

This yields $R=G-C, S=-E<0, s=C / 2>0$, and $r=G-C / 2-E$. In order to have fixed points in int $S_{4}$, we must have $0<E<G<C<$ $2(G-C)$. In this case $R+S+r+s=2(G-E)-C>0$. The fixed point on $W_{K}$ is a spiral sink for $K>1$ and a spiral source for $0<K<1$. This is an example with cyclic dynamics. 


\section{RECOMBINATION}

In this final section we modify the dynamics (1) by adding recombination:

$$
\dot{x}_{i}=x_{i}\left[(M \mathbf{x})_{i}-\bar{M}\right]-\varepsilon_{i} \rho D .
$$

Here $\varepsilon_{1}=-\varepsilon_{2}=-\varepsilon_{3}=\varepsilon_{4}=1, \rho>0$ is the recombination fraction and $D=x_{1} x_{4}-x_{2} x_{3}$ the linkage disequilibrium. This system on $S_{4}$ describes a haploid population (not quite realistic in view of the previous example, but in the tradition of game dynamical models; see Maynard Smith (1982)) which reproduces sexually, thereby giving rise to recombination. The two loci correspond to the two possible roles and the two alleles determine the strategy played in that role.

Then for $Z=x_{1} x_{4} / x_{2} x_{3}$ we have

$$
\frac{\dot{Z}}{Z}=\sum_{i=1}^{4} \varepsilon_{i} \frac{\dot{x}_{i}}{x_{i}}=-\rho D\left(\sum_{i=1}^{4} \frac{1}{x_{i}}\right)=-\rho(Z-1) x_{2} x_{3}\left(\sum_{i=1}^{4} \frac{1}{x_{i}}\right)
$$

which shows that $Z \rightarrow 1$ along all solutions in int $S_{4}$. Hence the Wright manifold $W_{1}=\{D=0\}=\{Z=1\}$ is globally attracting for the system (3), while the other manifolds $W_{K}$ are no longer invariant.

The dynamics on $W_{1}$ is the same as before and its expression can be simplified further: If $x=x_{1}+x_{3}$ denotes the frequency of $\mathbf{e}_{1}$ and $y=x_{1}+x_{2}$ the frequency of $f_{1}$, then on $W_{1}$ we have $x_{1}=x y, x_{2}=(1-x) y, \ldots$ and therefore

$$
\begin{aligned}
& \dot{x}=x(1-x)(S-(R+S) y) \\
& \dot{y}=y(1-y)(s-(r+s) x) .
\end{aligned}
$$

Hence the dynamics on $W_{1}$ coincide with the dynamics for bimatrix games introduced by Schuster and Sigmund (1981) and studied further in Schuster et al. (1981) and Hofbauer and Sigmund (1988, Chaps. 17 and 27).

\section{APPENDIX}

Using the transformation int $S_{4} \rightarrow$ int $\mathbb{R}_{+}^{3}, \mathbf{x} \mapsto \mathbf{y}$, where $y_{i}=x_{i} / x_{4}$, $i=1, \ldots, 4$ we write the replicator equation (1) as Lotka-Volterra equation

$$
\begin{aligned}
& \dot{y}_{1}=y_{1}\left((S+s)-(R+r) y_{1}-(R-s) y_{2}+(S-r) y_{3}\right) \\
& \dot{y}_{2}=y_{2}\left(s-r y_{1}+s y_{2}-r y_{3}\right) \\
& \dot{y}_{3}=y_{3}\left(S-R y_{1}-R y_{2}+S y_{3}\right) .
\end{aligned}
$$


The line of fixed points is given by the equations $r y_{1}-s y_{2}+r y_{3}=s$ and $R y_{1}+R y_{2}-S y_{3}=S . \quad H=\log \left(y_{1} / y_{2} y_{3}\right)$ is an invariant of motion, i.e., $y_{1}=K y_{2} y_{3}(K>0)$ define invariant surfaces $\widetilde{W}_{K}$ in int $\mathbb{R}_{+}^{3}$.

Therefore we can study the dynamics on these surfaces and reduce the system to two dimensions:

$$
\begin{aligned}
& \dot{y}_{2}=y_{2}\left(s+s y_{2}-r y_{3}-r K y_{2} y_{3}\right) \\
& \dot{y}_{3}=y_{3}\left(S-R y_{2}+S y_{3}-R K y_{2} y_{3}\right) .
\end{aligned}
$$

For $K=1$ we obtain

$$
\begin{aligned}
& \dot{y}_{2}=y_{2}\left(1+y_{2}\right)\left(s-r y_{3}\right) \\
& \dot{y}_{3}=y_{3}\left(1+y_{3}\right)\left(S-R y_{2}\right) .
\end{aligned}
$$

Hence the fixed point on $\tilde{W}_{1}$ is given by $\mathbf{F}=(S s / R r, S / R, s / r)$ (from now on $R r \neq 0$ ) and the line of fixed points can be written as

$$
\begin{aligned}
& y_{1}=\frac{S s}{R r}+(S s-R r) \lambda \\
& y_{2}=\frac{S}{R}+r(R+S) \lambda \\
& y_{3}=\frac{s}{r}+R(r+s) \lambda .
\end{aligned}
$$

This line intersects int $\mathbb{R}_{+}^{3}$ iff $R S>0$ and $r s>0$.

The Jacobian of (A1) at the fixed point $\mathbf{F}=\left(\hat{y}_{2}, \hat{y}_{3}\right)$ is given by

$$
J=\left(\begin{array}{cc}
\left(s-r K \hat{y}_{3}\right) \hat{y}_{2} & -r\left(1+K \hat{y}_{2}\right) \hat{y}_{2} \\
-R\left(1+K \hat{y}_{3}\right) \hat{y}_{3} & \left(S-R K \hat{y}_{2}\right) \hat{y}_{3}
\end{array}\right) .
$$

We note that

$$
K=\frac{S s / R r+(S s-R r) \lambda}{(S / R+r(R+S) \lambda)(s / r+R(r+s) \lambda)} .
$$

A direct computation yields

$$
\operatorname{tr} J=R r(R+S+r+s) \lambda
$$

and

$$
\operatorname{det} J=-(R+S)(r+s)\left[R r(S s-R r) \lambda^{2}+2 S s \lambda+S s / R r\right] .
$$


It is easy to check that det $J$ does not change its sign along the line of fixed points.

Equation (A1) can be written as

$$
\begin{aligned}
& \dot{y}_{2}=y_{2}\left[s\left(1+y_{2}+y_{3}+K y_{2} y_{3}\right)-(r+s)\left(1+K y_{2}\right) y_{3}\right] \\
& \dot{y}_{3}=y_{3}\left[S\left(1+y_{2}+y_{3}+K y_{2} y_{3}\right)-(R+S)\left(1+K y_{3}\right) y_{2}\right] .
\end{aligned}
$$

After dividing the right-hand side by $y_{2} y_{3}\left(1+y_{2}+y_{3}+K y_{2} y_{3}\right)$-which does not change the orbits-we obtain

$$
\begin{aligned}
& \dot{y}_{2}=\frac{s}{y_{3}}-\frac{(r+s)\left(1+K y_{2}\right)}{1+y_{2}+y_{3}+K y_{2} y_{3}} \\
& \dot{y}_{3}=\frac{S}{y_{2}}-\frac{(R+S)\left(1+K y_{3}\right)}{1+y_{2}+y_{3}+K y_{2} y_{3}} .
\end{aligned}
$$

The divergence of this system is given by

$$
\operatorname{div}\left(\dot{y}_{2}, \dot{y}_{3}\right)=-\frac{(R+S+r+s)(K-1)}{\left(1+y_{2}+y_{3}+K y_{2} y_{3}\right)^{2}}
$$

If $K=1(\Leftrightarrow \lambda=0)$ or $R+S+r+s=0$ (these are the cases for which $\operatorname{tr} J=0$ ) the system is divergence-free and hence is Hamiltonian, i.e., of the form $\dot{y}_{2}=-\partial H / \partial y_{3}, \dot{y}_{3}=\partial H / \partial y_{2}$. The Hamilton function $H$ can be easily computed to:

$$
H=S \log y_{2}-(R+S) \log \left(1+y_{2}\right)-s \log y_{3}+(r+s) \log \left(1+y_{3}\right)
$$

in the case $K=1$; in the case $R+S+r+s=0$ it is given by

$$
H=S \log y_{2}-s \log y_{3}+(r+s) \log \left(1+y_{2}+y_{3}+K y_{2} y_{3}\right) .
$$

We note that $H$ is a constant of motion. Therefore the fixed point is either a saddle or surrounded by periodic orbits.

If $K \neq 1$ and $R+S+r+s \neq 0$ then $\operatorname{div}\left(\dot{y}_{2}, \dot{y}_{3}\right)$ is strictly positive or strictly negative, having the same sign as $\operatorname{tr} J$. Hence--by the Theorem of Bendixson-Dulac--there does not exist any periodic orbit on $\tilde{W}_{K}$.

The following classification of the fixed points in int $S_{4}$ characterises also the global dynamics on each surface $\widetilde{W}_{K}$ :

If $R r>0$ then det $J<0$ and all fixed points are saddles.

If $R r<0$ then $\operatorname{det} J>0$ and we obtain:

\begin{tabular}{cccc} 
& $K<1$ & $K=1$ & $K>1$ \\
\hline$R+S+r+s<0$ & Sink & Centre & Source \\
$R+S+r+s=0$ & Centre & Centre & Centre \\
$R+S+r+s>0$ & Source & Centre & Sink
\end{tabular}




\section{ACKNOWLEDGMENTS}

We thank R. Gardner and A. J. Metz for stimulating conversations. Support from the Austrian Fonds zur Förderung der wissenschaftlichen Forschung Project P6668 is gratefully acknowledged.

\section{REFERENCES}

Crow, J. F., and Kimura, M. 1970. "An Introduction to Population Genetics Theory," Harper and Row, New York.

Dawkins, R. 1976. "The Selfish Gene," Oxford Univ. Press, London/New York.

FARKAS, M. 1984. Zip bifurcations in a competition model, Nonlinear Anal. TMA 8, 1295-1309.

GARDNER, R., AND MORRIS, M. 1989. The evolution of bluffing in animal contests: An ESS approach, J. Theor. Biol. 137, 235-243.

Hammerstein, P. 1981. The role of asymmetries in animal contests, Anim. Behav. 29, 193-205.

Hofbauer, J., ANd Sigmund, K. 1988. "The Theory of Evolution and Dynamical Systems," Cambridge Univ. Press, Cambridge.

MaYnard Smith, J. 1982. "Evolution and the Theory of Games," Cambridge Univ. Press, Cambridge.

Maynard Smith, J., and Parker, G. 1976. The logic of asymmetric contests, Anim. Behav. 24, 159-179.

RIECHERT, S. 1978. Games spiders play: Behavioural variability in territorial disputes, Behav. Ecol. Sociobiol. 3, 135-162.

SFLTFN, R. 1980. A note on evolutionarily stable strategies in asymmetrical animal conflicts, J. Theor. Biol. 84, 93-101.

Schuster, P., AND Sigmund, K. 1981. Coyness, philandering and stable strategies, Anim. Behav. 29, 186-192.

Schuster, P., Sigmund, K., Hofbauer, J., ANd WolfF, R. 1981. Selfregulation of behaviour in animal societies. II. Games between two populations without selfinteraction, Biol. Cybern. 40, 9-15.

TAYLOR, P. 1979. Evolutionarily stable strategies with two types of players, J. Appl. Prob. 16, 76-83.

TAYLOR, P., AND JONKER, L. 1978. Evolutionarily stable strategies and game dynamics, Math. Biosci. 40, 145-156. 